\title{
Stabilization by vertical vibrations
}

\author{
Daniel Núñez \\ Departamento de Matemática, \\ Universidad del Zulia, Maracaibo, 4001, Venezuela \\ Pedro J. Torres* \\ Departamento de Matemática Aplicada, \\ Universidad de Granada, 18071 Granada, Spain.
}

\begin{abstract}
We consider a general version of the classical problem of the stabilization of the inverted pendulum by a vertical periodic vibration of the point of suspension. Instead of the usual harmonic motion, we propose an oscillatory control with a piecewise constant acceleration, obtaining explicit conditions over the frequency or the amplitude leading to the desired stabilization. Keywords: Stability, inverted pendulum, vibrational control, twist. AMS Subject Classification: 34D20, 34C15, 37J25, 37J40
\end{abstract}

\section{Introduction}

Vibrational stabilization is a broad field with interesting applications to different problems in the applied sciences. In Quantum Mechanics, it is employed to implement some trapping mechanisms for elementary particles like the Paul trap $[4,16]$. In Mechanical Engineering, it is a fundamental concept in the control of robotic devices $[5,13]$. The book [2] presents a comprehensive survey on Vibrational Mechanics, including many applications. Among them, the stabilization of an inverted pendulum by vertical vibrations of the point of suspension is a classical problem that has become a paradigm in Dynamical Systems and Control Theory. It is worth to mention that the problem of stabilizing an inverted pendulum is reaching the centenary, since the first references go back to Stephenson in 1908 [19, 20]. Later, Kapitza [6] renewed the interest in this problem and after this contribution the number of related articles is huge and cover many different approaches. From the mathematical point of view, most of the works study the linearized problem $[3,7,8]$. When the oscillation of the

*Supported by projects MTM2005-03483 and FQM2216. 
axis is harmonic, a first rigorous proof of stability in the nonlinear sense was done in [1].

A rigid pendulum can be seen as a particle sliding over a circumference under the only action of gravity, therefore the problem of the stabilization of the inverted pendulum can be seen as the search of a suitable parametric excitation of the circumference for which the upper equilibrium becomes stable. More generally, one can imagine the motion of a particle constrained to a pulsating curve under the only action of gravity and ask about the stability properties of relative equilibria. This general problem was raised in [12]. Some first results were obtained concerning pulsating elliptic and quartic curves. As an open problem, it was pointed out the possibility of stabilizing the maximum position of a plane curve that is symmetric with respect to a vertical line, in analogy to the inverted pendulum (see Picture 1).
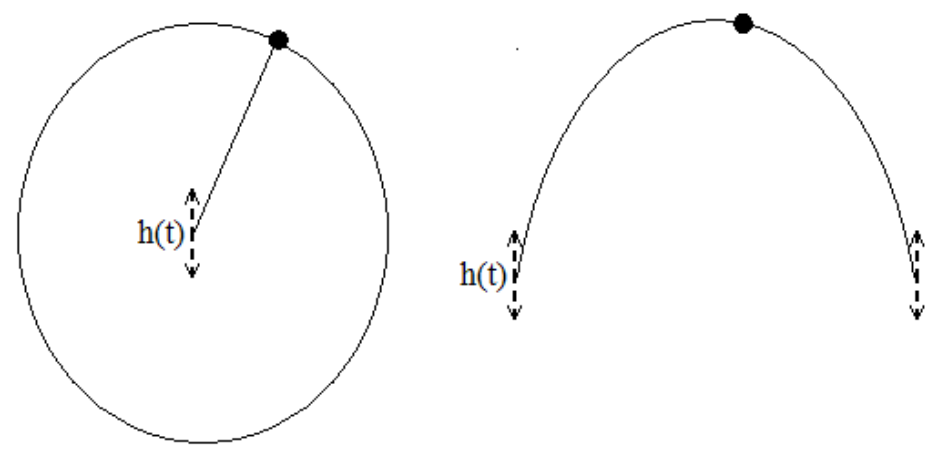

Figure 1: The inverted pendulum and the stabilization of a maximal equilibrium.

Mathematically, the problem is described as follows. Let us consider a plane and smooth curve parametrized by the arc length

$$
\alpha(s)=(X(s), Y(s)), s \in \mathbb{R}
$$

such that $X$ is an odd function and $Y$ is an even function. Under such conditions, the curve is symmetric with respect to $s=0$. Our purpose is to study the movement of a bead sliding on this curve under the only action of gravity when the equilibrium point $s=0$ is a maximum with negative curvature $Y^{\prime \prime}(0)<0$. The rest point $s=0$ is unstable and the problem consists in finding a periodic vertical movement $h(t)$ of this curve in order to stabilize this equilibrium. Note that the simplest case $\alpha(s)=(\sin s, \cos s)$ corresponds to the inverted pendulum.

We add a vertical movement making

$$
\alpha(t, s)=(X(s), Y(s)+h(t)), t, s \in \mathbb{R}
$$


where $h(t)$ is an adequate $T$-periodic function or vibrational control. By an adequate renormalization, the gravitational constant and the mass are fixed as 1 , and therefore the potential energy is $U(t, s)=Y(s)+h(t)$ and the lagrangian can be written as

$$
L(t, s, \dot{s})=\frac{1}{2} \dot{s}^{2}+\left(\alpha_{t}(t, s) \cdot \alpha_{s}(t, s)\right) \dot{s}+\frac{1}{2} \alpha_{t}(t, s) \cdot \alpha_{t}(t, s)-U(t, s) .
$$

Consequently, the Euler-Lagrange motion's equation is (see [12] for details)

$$
s^{\prime \prime}+\left(\alpha_{t t}(t, s)+e_{2}\right) \cdot \alpha_{s}(t, s)=0
$$

where $e_{2}=(0,1)$ and $\cdot$ is the usual scalar product, or in a simplified form

$$
s^{\prime \prime}+Y^{\prime}(s)\left(1+h^{\prime \prime}(t)\right)=0 .
$$

Notice that $s=0$ is still an equilibrium for this equation because of the oddness of $Y^{\prime}(s)$. The objective is to find adequate vibrational controls $h(t)$ such that the equilibrium $s=0$ becomes stable in the sense of Lyapunov.

We propose two different strategies: high-frequency vs modulated-amplitude vibrations. Both cases are constructed from the basic function

$$
p(t)= \begin{cases}\frac{1}{2} t(\pi-t) & \text { if } 0 \leq t<\pi \\ \frac{1}{2}(t-\pi)(t-2 \pi) & \text { if } \pi \leq t<2 \pi\end{cases}
$$

This control function is of class $C^{1}$ and the second derivative is piecewise constant (see Picture 2), which is a key feature in order to simplify the computations.
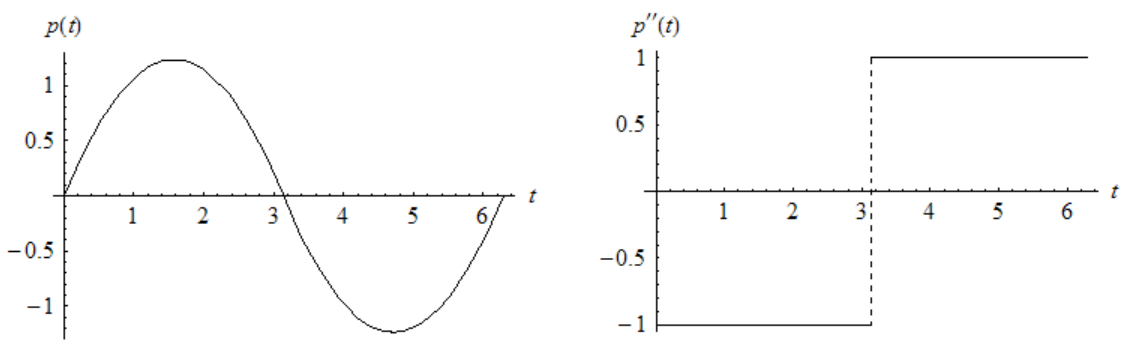

Figure 2: The vibrational control $p(t)$ and its second derivative.

Section 2 is devoted to the stabilization through high-frequency oscillations. For a certain range of curvatures at the equilibrium point, it is obtained a numerically computable frequency such that stabilization holds for higher frequencies. This generalizes the classical results on the inverted pendulum. The explicit estimation of the critical frequency is a remarkable advantage if it is compared with other approaches of perturbative type like averaging theory [5].

In Section 3, we study the stabilizing effect at a fixed frequency of the control taking its amplitude as the relevant parameter. It is found that the equilibrium 
point is stable in the nonlinear sense provided that the amplitude belongs to a given set $\Lambda$ (depending on the curvature), which is composed by a sequence of disjoint open intervals tending $+\infty$. This set is also numerically computable.

With respect to the method of proof, after an analysis of the linear stability, it is shown that the first twist coefficient is different from zero. This implies the existence of a foliation of invariant KAM tori in a neighborhood of the equilibrium, which in turn gives the stability in the nonlinear sense. We use an explicit formula for the twist coefficient obtained in $[14,15]$ and refined in [9]. Besides the yet mentioned explicit estimates of the parameters, other advantage of our method is that the control is robust, that is, a small perturbation of the vertical vibration keeps the stabilizing effect. On the contrary, it is not possible to give a quantitative estimate of the stability region around the equilibrium, which is an interesting open problem.

From now on, it is assumed that the curvature $Y^{\prime \prime}(0)$ at the rest point is negative and we denote $k^{2}=\left|Y^{\prime \prime}(0)\right|$.

\section{High-frequency oscillatory control.}

In this section, we explore the stabilizing effect of a vertical vibration $p(w t)$, where $p$ is defined by (3), for high values of the frequency $w$. The resulting equation is

$$
s^{\prime \prime}+Y^{\prime}(s)\left(1+w^{2} p^{\prime \prime}(w t)\right)=0 .
$$

\subsection{Linear stability}

The linearized or variational equation of (2) around $s=0$ is the Hill's equation

$$
x^{\prime \prime}+a(t) x=0,
$$

where $a(t)$ is a piecewise-constant periodic function given by

$$
a(t)=Y^{\prime \prime}(0)\left(1+w^{2} p^{\prime \prime}(w t)\right)= \begin{cases}k^{2}\left(w^{2}-1\right) & \text { if } 0<t<\frac{\pi}{w} \\ -k^{2}\left(w^{2}+1\right) & \text { if } \frac{\pi}{w}<t<\frac{2 \pi}{w} .\end{cases}
$$

Note that the mean value of $a$ over a whole period is negative, a case which is not covered by the variety of stability criteria for the Hill's equation available in the literature (see $[11,18,21])$. This particularity leads to a direct integration in order to compute the discriminant.

In the following, we assume $w>1$. Then, a tedious but elemental piecewise integration of the equation gives the monodromy matrix

$$
M=\left(\begin{array}{cc}
\cosh B \cos A-A / B \sinh B \sin A & \frac{\pi}{w A} \cosh B \sin A+\frac{\pi}{w B} \sinh B \cos A \\
\frac{w B}{\pi} \cos A \sinh B-\frac{w A}{\pi} \cosh B \sin A & B / A \sinh B \sin A+\cosh B \cos A
\end{array}\right)
$$


with

$$
\begin{aligned}
& A \equiv A(w, k)=\frac{\pi k \sqrt{w^{2}-1}}{w} \\
& B \equiv B(w, k)=\frac{\pi k \sqrt{w^{2}+1}}{w} .
\end{aligned}
$$

Therefore, the discriminant is

$$
\Delta(w, k)=2 \cosh B \cos A-\left[\frac{B}{A}-\frac{A}{B}\right] \sinh B \sin A .
$$

The main result of this section is the following one.

Proposition 1 Let us define the set

$$
\mathcal{K}=\{k>0:|\cosh (\pi k) \cos (\pi k)|<1\}
$$

and assume that $k \in \mathcal{K}$. Then, there exists a critical frequency $w_{0}(k)>0$ such that (5) is stable for all $w>w_{0}(k)$.

Proof. An elemental computation gives

$$
\lim _{w \rightarrow+\infty} A(w, k)=\pi k, \quad \lim _{w \rightarrow+\infty} B(w, k)=\pi k,
$$

and then

$$
\lim _{w \rightarrow+\infty} \Delta(w, k)=2 \cosh (\pi k) \cos (\pi k) .
$$

It is well-known (see [11] for details) that $|\Delta|<2$ is a sufficient condition for the stability of a given Hill's equation. The result follows trivially.

Remark 1 The set $\mathcal{K}$ is composed by a sequence of open intervals $I_{n}$ with $n+$ $\frac{1}{2} \in I_{n}$ for all $n \in \mathbb{N}$. A numerical computation gives

$$
\mathcal{K}=] 0,0.596864[\cup] 1.4918,1.50562[\cup] 2.44975,2.50025[\ldots
$$

Remark 2 The critical frequency can be explicitly given by

$$
w_{0}(k)=\sup \{w>1:|\Delta(w, k)| \geq 2\}
$$

As it was mentioned in the Introduction, the critical frequency $w_{0}$ is numerically computable, due to the global character of our arguments. For instance, $w_{0}(0.5)=1, w_{0}(0.55)=1.12646, w_{0}(0.59)=3.21142, w_{0}(0.59686)=132.285$ and diverges to $+\infty$ as $k$ approaches the upper limit of the first interval of $\mathcal{K}$. For higher curvatures, the critical frequency increases drastically, $w_{0}(5.5)=11042$, $w_{0}(7.5)=303547$. 


\subsection{Nonlinear stability}

The system (4) under study is conservative, so the stability in the sense of Lyapunov can not be directly derived from the first approximation because of the possible synchronized influence of higher terms leading to resonance. After the works of Moser [17], it is well known that the stability in the nonlinear sense depends generically on the third approximation around $s=0$

$$
s^{\prime \prime}+a(t) s+c(t) s^{3}=0,
$$

with

$$
a(t)=Y^{\prime \prime}(0)\left(1+w^{2} p^{\prime \prime}(w t)\right), \quad c(t)=\frac{1}{6} Y^{\prime \prime \prime \prime}(0)\left(1+w^{2} p^{\prime \prime}(w t)\right) .
$$

In [14], Ortega proves that if the equilibrium $s=0$ is stable for the linearized equation

$$
s^{\prime \prime}+a(t) s=0,
$$

then it is stable in the nonlinear sense provided the first twist coefficient is different from zero. In particular it occurs when $c(t)$ does not changes sign (main result of [14])

The proof is based on the twist theorem of Moser combined to the Birkhoff Normal Form of the Poincaré map associated to (4). Next we shall explain it more explicitly for the reader's convenience. First we make some definitions.

Let $M$ be the monodromy matrix of (11). The eigenvalues $\lambda_{1,2}$ of $M$ are called the Floquet multipliers of (11). It is well known that the Floquet multipliers of (11) satisfy

$$
\lambda_{1} \lambda_{2}=1 .
$$

In a classical terminology, it is said that (11) (or $s=0$ ) is elliptic if $\lambda_{2}=\overline{\lambda_{1}} \epsilon$ $\mathbb{C}^{1} \backslash\{ \pm 1\}$, parabolic if $\lambda_{1,2}= \pm 1$ and hyperbolic if $\left|\lambda_{1,2}\right| \neq 1$ respectively. In the hyperbolic case not only the linear equation is unstable but also $s=0$ like solution of (4).

Given $n \in \mathbb{N}$ we say that the equilibrium $s \equiv 0$ of (4) is $n$-resonant if it is elliptic and the Floquet multipliers satisfy $\lambda_{i}^{n}=1$. We say that $s=0$ is strongly resonant if it is $n$-resonant for $n=3$ or 4 .

The Poincaré mapping associated to (4) is defined near the origin by

$$
\mathcal{P}(x, y)=\varphi(T ; x, y),
$$

where $\varphi(t ; x, y)$ is the unique solution of $(9)$ such that $\varphi(t ; x, y)=x, \varphi^{\prime}(0 ; x, y)=$ $y$, and $T=\frac{2 \pi}{\omega}$ is the minimal period of (4).

Note that $\mathcal{P}(0,0)=(0,0)$ and then the stability of $s \equiv 0$ for (4) is equivalent to the stability of $(0,0)$ as fixed point of $\mathcal{P}$. Other elementary property of the Poincaré map states that $\mathcal{P}^{\prime}(0,0)$ is a monodromy matrix for $(11)$, then its eigenvalues are the Floquet multipliers of (11). If (11) is elliptic and not strongly resonant, the Birkhoff's normal form Theorem provides a canonical change of 
variables $z=\Phi(\xi), z=(x, y)$, such that $\mathcal{P}$ adopts in the new coordinates the following form

$$
\mathcal{P}^{*}(\xi)=\left(\Phi^{-1} \circ \mathcal{P} \circ \Phi\right)(\xi)=R\left[\theta+\beta|\xi|^{2}\right](\xi)+O_{4},
$$

where $R[\delta]$ denotes the rotation of angle $\delta, \lambda=e^{ \pm i \theta}$ are the Floquet multipliers, and $O_{4}$ indicates a term that is $O\left(|\xi|^{4}\right)$ when $\xi \rightarrow 0$. The coefficient $\beta$ is called the first twist coefficient and play a central role in the stability. From the Twist Theorem it follows that if $\beta \neq 0$ then $(0,0)$ is stable (see chapter 3 of [17]).

On the other hand, the reason of excluding the 3-resonant case in the general process of the Birkhoff normal form is to eliminate the second order terms of $\mathcal{P}$ by a suitable canonical change of variables. In a similar way one must exclude the 4-resonant case to simplify the terms of third order of $\mathcal{P}$. However, in our case $\mathcal{P}$ has not any terms of second order (see (9)), then it is sufficient to exclude the 4-resonant case in our study. In other words if (11) is elliptic and not 4resonant then we can define the twist coefficient $\beta$ associated to (4) by means (13).

The twist coefficient has an explicit formula and it is proportional to the integral quantity (see $[14,15]$ )

$$
\beta=\int_{0}^{\frac{2 \pi}{w}} r(t)^{4} c(t) d t
$$

where $r(t)$ is the modulus of the unique complex solution $\Psi(t)$ of (11) with initial conditions $\Psi(0)=1$ and $\Psi^{\prime}(0)=i$. In [9] the authors pointed out an interesting connection between the Hill's equation and the Emarkov-Pinney equation. More precisely, in Sections 3.2 and 3.4 of the mentioned paper, the authors show that if (11) is elliptic then $r(t)$ is the unique $T$-periodic positive solution of the singular equation

$$
r^{\prime \prime}+a(t) r=\frac{1}{r^{3}} .
$$

This characterization of $r(t)$ will be a key fact in the proofs.

Now we are in the position to state and prove the main result of the paper.

Theorem 1 Let us assume that the discriminant defined by (8) verifies $0<$ $|\Delta(w, k)|<2$. If $Y^{\prime \prime \prime \prime}(0) \neq 0$, then the equilibrium $s=0$ of $(4)$ is stable in the nonlinear sense.

Proof. By the condition imposed on the discriminant, the equilibrium is elliptic and not 4-resonant. So only it remains to prove that $\beta \neq 0$. Note that

$$
\beta=\frac{1}{6} Y^{\prime \prime \prime \prime}(0) \int_{0}^{\frac{2 \pi}{w}} r(t)^{4}\left(1+w^{2} p^{\prime \prime}(w t)\right) d t
$$

and due to the hypothesis $Y^{\prime \prime \prime \prime}(0) \neq 0$, we can operate on the proportional coefficient

$$
\beta^{*}=\int_{0}^{\frac{2 \pi}{w}} r(t)^{4}\left(1+w^{2} p^{\prime \prime}(w t)\right) d t=\left(1-w^{2}\right) \int_{0}^{\frac{\pi}{w}} r(t)^{4} d t+\left(1+w^{2}\right) \int_{\frac{\pi}{w}}^{\frac{2 \pi}{w}} r(t)^{4} d t .
$$


We are going to prove that this quantity is negative.

Let us remember that $r$ is the unique positive $\frac{2 \pi}{w}$-periodic solution of the singular equation (15). On the interval $\left[0, \frac{\pi}{w}\right], r$ verifies the autonomous equation

$$
r^{\prime \prime}+k\left(w^{2}-1\right) r=\frac{1}{r^{3}} .
$$

Multiplying by $r^{3}$ and integrating over $\left[0, \frac{\pi}{w}\right]$ it results

$$
k\left(w^{2}-1\right) \int_{0}^{\frac{\pi}{w}} r(t)^{4} d t=\frac{\pi}{w}-r\left(\frac{\pi}{w}\right)^{3} r^{\prime}\left(\frac{\pi}{w}\right)+r(0)^{3} r^{\prime}(0)+3 \int_{0}^{\frac{\pi}{w}}\left[r(t) r^{\prime}(t)\right]^{2} d t
$$

after a trivial integration by parts. On the other hand, on the interval $\left[\frac{\pi}{w}, \frac{2 \pi}{w}\right]$, the corresponding equation for $r$ is

$$
r^{\prime \prime}-k\left(w^{2}+1\right) r=\frac{1}{r^{3}}
$$

and operating analogously one obtains

$k\left(w^{2}+1\right) \int_{\frac{\pi}{w}}^{\frac{2 \pi}{w}} r(t)^{4} d t=-\frac{\pi}{w}+r\left(\frac{2 \pi}{w}\right)^{3} r^{\prime}\left(\frac{2 \pi}{w}\right)-r\left(\frac{\pi}{w}\right)^{3} r^{\prime}\left(\frac{\pi}{w}\right)-3 \int_{\frac{\pi}{w}}^{\frac{2 \pi}{w}}\left[r(t) r^{\prime}(t)\right]^{2} d t$.

Therefore, by introducing this information on $\beta^{*}$ and using the periodicity of $r$, one obtains

$$
\beta^{*}=-\frac{1}{k}\left[\frac{2 \pi}{w}+3 \int_{0}^{\frac{2 \pi}{w}}\left[r(t) r^{\prime}(t)\right]^{2} d t\right]<0
$$

and the proof is complete.

Corollary 1 Let us assume that $k \in \mathcal{K}$, with $k \neq n+\frac{1}{2}, \forall n \in \mathbb{N} \cup\{0\}$, and $Y^{\prime \prime \prime \prime}(0) \neq 0$. Then, there exists a critical frequency $w_{0}(k)>0$ such that the equilibrium $s=0$ of (4) is stable in the nonlinear sense for all $w>w_{0}(k)$.

Proof. By the conditions imposed over $k$, we have that

$$
0<\left|\lim _{w \rightarrow+\infty} \Delta(w, k)\right|<2 .
$$

Therefore, by continuity we are in the conditions of the latter theorem for high values of $w$.

Remark 3 We emphasize once again that, since the discriminant $\Delta(w, k)$ is explicitly known, it is very easy to perform numerical computations in order to obtain explicit values of $w_{0}(k)$.

Remark 4 In the case or an inverted pendulum of length $L$, the corresponding vibrating curve is a circumference of curvature $\frac{1}{L}$, that is, $k=\frac{1}{\sqrt{L}}$. The stabilization is achieved for high frequencies if $k \in \mathcal{K}$, which corresponds to large values of the length $L$ (then $k \in I_{1}$ ), while there are some gaps in the values of $L$ as it approaches to zero. Moreover, from the numerical results, smaller pendulum's lengths require higher frequencies to get stabilization. In the next section, we will try to cover such gaps with a different control. 


\section{Modulated-amplitude oscillatory control.}

In this section we consider the stabilizing effect of a vertical vibration of fixed frequency by modulating its amplitude. The periodic control is $\lambda p(t)$, where $\lambda$ is a parameter which represent the magnitude or amplitude os the oscillation. In this case, the equation is

$$
s^{\prime \prime}+Y^{\prime}(s)\left(1+\lambda p^{\prime \prime}(t)\right)=0
$$

\subsection{Linear stability}

In this case, the variational equation of (16) around $s=0$ is the Hill's equation

$$
x^{\prime \prime}+a(t) x=0
$$

where $a(t)$ is a piecewise-constant periodic function given by

$$
a(t)=Y^{\prime \prime}(0)\left(1+\lambda p^{\prime \prime}(t)\right)= \begin{cases}k^{2}(\lambda-1) & \text { if } 0<t<\pi \\ -k^{2}(\lambda+1) & \text { if } \pi<t<2 \pi\end{cases}
$$

By assuming $\lambda>1$, the discriminant (that is, the trace of the monodromy matrix) is

$$
\Delta(\lambda, k)=2 \cosh D \cos C-\left[\frac{D}{C}-\frac{C}{D}\right] \sinh D \sin C .
$$

with

$$
\begin{gathered}
C \equiv C(\lambda, k)=\pi k \sqrt{\lambda-1} \\
D \equiv D(\lambda, k)=\pi k \sqrt{\lambda+1} .
\end{gathered}
$$

Now, the following result can be proved.

Proposition 2 For every $k$, let us define the open set

$$
\Lambda=\left\{\lambda \in \mathbb{R}^{+}:|\Delta(\lambda, k)|<2\right\}
$$

for which (17) is stable. Then $\Lambda$ is composed by a sequence of disjoint open intervals tending to $+\infty$.

Proof. Let us fix the sequence $\lambda_{n}=\frac{n^{2}}{k^{2}}+1$. It is easy to verify that

$$
\lim _{n \rightarrow+\infty} \Delta\left(\lambda_{2 n}, k\right)=+\infty, \quad \lim _{n \rightarrow+\infty} \Delta\left(\lambda_{2 n+1}, k\right)=-\infty .
$$

Hence, by continuity, for any there exists a given $n_{0}$ such that for any $n>n_{0}$, there exists an open interval $\left.J_{n} \subset\right] n, n+1[$ such that $|\Delta(\lambda, k)|<2$ for every $\lambda \in J_{n}$. The proof ends by defining $\Lambda=\bigcup_{n>n_{0}} J_{n}$. 
Remark 5 For a given $k$, it is easy to find the set $\Lambda$ numerically. For $k=1$,

$$
\Lambda=] 2.925963,2.933335[\cup] 7.024295,7.025168[\cup \ldots
$$

For $k=0.1$,

$$
\Lambda=] 223.855,227.241[\cup] 625.623,625.868[\cup \ldots
$$

As it is seen, it is possible to stabilize for any curvature but the amplitude must be tuned up in a quite precise way.

\subsection{Nonlinear stability}

To study the stability in the nonlinear sense, we consider again the third-order approximation around $s=0$

$$
s^{\prime \prime}+a(t) s+c(t) s^{3}=0,
$$

with

$$
a(t)=Y^{\prime \prime}(0)\left(1+\lambda p^{\prime \prime}(t)\right), \quad c(t)=\frac{1}{6} Y^{\prime \prime \prime \prime}(0)\left(1+\lambda p^{\prime \prime}(t)\right) .
$$

For a given $k>0$, let us define the open set

$$
\Lambda_{0}=\left\{\lambda \in \mathbb{R}^{+}: 0<|\Delta(\lambda, k)|<2\right\} .
$$

This is just the set $\Lambda$ defined in Subsection 3.1 minus a sequence $\left\{\lambda_{n}\right\}_{n}$ for which the discriminant is zero. The main result of this section is the following theorem.

Theorem 2 Given an arbitrary $k>0$, let us assume that $\lambda \in \Lambda_{0}(k)$ and $Y^{\prime \prime \prime \prime}(0) \neq 0$. Then, the equilibrium $s=0$ of $(16)$ is stable in the nonlinear sense.

Proof. By Subsection 3.1, the equilibrium is linearly stable. Moreover, the discriminant is not zero so the equilibrium is not 4-resonant. The twist coefficient is proportional to

$$
\beta=\frac{1}{6} Y^{\prime \prime \prime \prime}(0) \int_{0}^{2 \pi} r(t)^{4}\left(1+\lambda p^{\prime \prime}(t)\right) d t .
$$

It remains to prove that $\beta \neq 0$, and the proof is analogous to that of Theorem 1 , so we omit it.

\section{References}

[1] B.S. Bardin, A.P.Markeyev, The stability of the equilibrium of a pendulum for vertical oscillations of the point of suspension, J. Appl. Maths. Mechs. 59 (1995), no. 6, 879-886. 
[2] I.I. Blekhman, "Vibrational Mechanics", World Scientific, 2000.

[3] H. W. Broer, I. Hoveijn, M. van Noort, The inverted pendulum: a singularity theory approach, J. Differential Equations 157 (1999), no. 1, 120-149.

[4] L.S. Brown, Quantum motion in a Paul trap, Phys.Rev.Lett. 66 (1991), n.5, 527-529.

[5] F. Bullo, Averaging and vibrational control of mechanical systems, SIAM J. Control Optim. 41 (2002), n. 2, 542-562.

[6] P. L. Kapitza, Dynamic stability of a pendulum with an oscillating point of suspension (in Russian), Zurnal Eksperimentalno j i Teoreticeskoj Fiziki 21(5) (1951), 588-597.

[7] M. Levi, Stability of the inverted pendulum - a topological explanation, SIAM Rev. 30 (1988), no. 4, 639-644.

[8] M. Levi and W. Weckesser, Stabilization of the inverted linearized pendulum by high frequency vibrations, SIAM Rev. 37 (1995), no. 2, 219-223.

[9] J. Lei, X. Li, P. Yan, M. Zhang, Twist character of the least amplitude periodic solution of the forced pendulum, SIAM J. Math. Anal., 35(2003), pp. $844-867$.

[10] B. Liu, The stability of the equilibrium of a conservative system, J. Math. Anal. Appl. 202 (1996), 133-149.

[11] W. Magnus, S. Winkler, "Hill's Equation", Dover, New York, 1979

[12] D. Núñez, P.J. Torres, KAM dynamics and stabilization of a particle sliding over a periodically driven wire, Appl. Math. Lett. 20 (2997), 610-615.

[13] Y. Nakamura, T. Suzuki, M. Koimura, Nonlinear behavior and control of a nonholonomic free-joint manipulator, IEEE Trans. Robotics and Automation 13 (1997), 853-862.

[14] R. Ortega, The stability of the equilibrium of a nonlinear Hill's equation, SIAM J. Math. Anal. 25 (1994), 1393-1401.

[15] R. Ortega, Periodic solutions of a newtonian equation: stability by the third approximation, J. Diff. Equ. 128 (1996), 491-518.

[16] W Paul, Electromagnetic traps for charged and neutral particles, Rev. Mod. Phys. 62 (1990), n.3, 531-540.

[17] C. L. Siegel and J. K. Moser, "Lectures on Celestial Mechanics", SpringerVerlag, New York. Berlin, 1971.

[18] V.M. Starzinskii, Survey of works on conditions of stability of the trivial solution of a system of linear differential equations with periodic coefficients, in Amer. Math. Soc. Transl. Ser. 2, Vol.1, Providence, RI, 1955. 
[19] A. Stephenson, On a new type of dynamic stability, Memoirs and Proceedings of the Manchester Literary and Philosophical Society 52(8) (1908), $1-10$.

[20] A. Stephenson, On induced stability, Philosophical Magazine 15 (1908), 233-236.

[21] M. Zhang and W. Li, A Lyapunov-Type stability criterion using $L^{\alpha}$ norms, Proc. Amer. Math. Soc., 130 (2002), n. 11, 3325-3333. 